\title{
dc and ac transport in few-layer black phosphorus
}

Cite as: J. Appl. Phys. 127, 044302 (2020); https://doi.org/10.1063/1.5129273

Submitted: 26 September 2019 . Accepted: 08 January 2020 . Published Online: 22 January 2020

M. Tahir (D. P. M. Krstajić, and P. Vasilopoulos
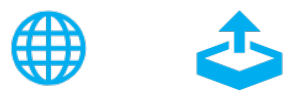

Export Citation

CrossMark

\section{ARTICLES YOU MAY BE INTERESTED IN}

Nanoscale electro-thermal interactions in AlGaN/GaN high electron mobility transistors

Journal of Applied Physics 127, 044502 (2020); https://doi.org/10.1063/1.5123726

Persistent and reversible electrostatic control of doping in graphene/hexagonal boron nitride heterostructures

Journal of Applied Physics 127, 044303 (2020); https://doi.org/10.1063/1.5127770

Doping induced charge density wave in monolayer $\mathrm{TiS}_{2}$ and phonon-mediated superconductivity

Journal of Applied Physics 127, 044301 (2020); https://doi.org/10.1063/1.5135615

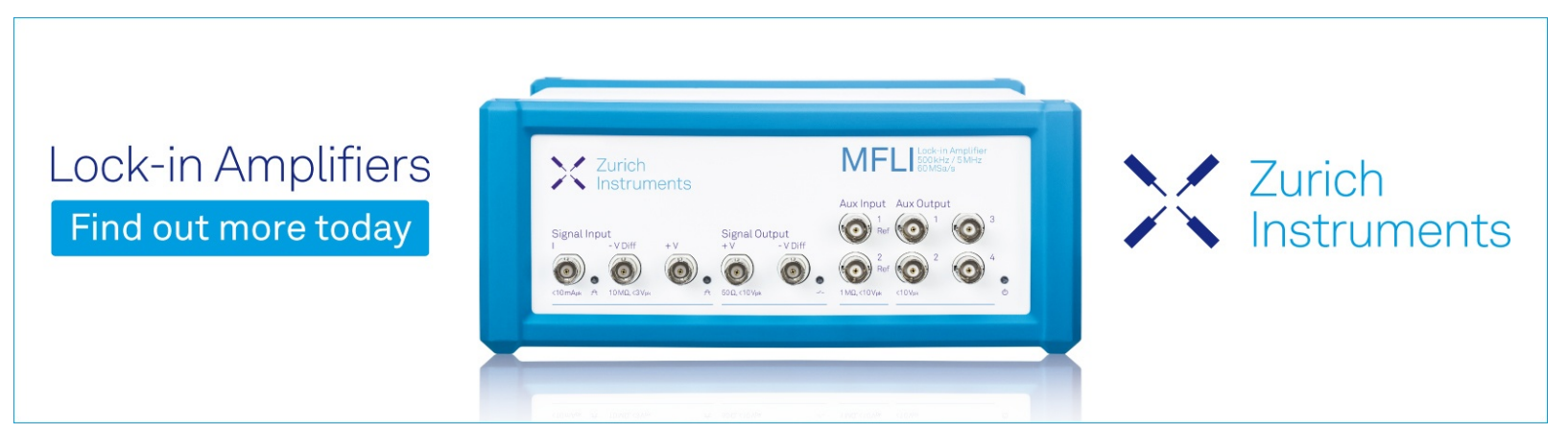




\title{
dc and ac transport in few-layer black phosphorus
}

Cite as: J. Appl. Phys. 127, 044302 (2020); doi: 10.1063/1.5129273

Submitted: 26 September 2019 . Accepted: 8 January 2020 .

Published Online: 22 January 2020

M. Tahir, ${ }^{1, a)}$ (D) P. M. Krstajić, ${ }^{2, b)}$ and P. Vasilopoulos ${ }^{3, c)}$

\author{
AFFILIATIONS \\ ${ }^{1}$ Department of Physics, Colorado State University, Fort Collins, Colorado 80523, USA \\ ${ }^{2}$ Center of Microelectronic Technologies, ICTM, University of Belgrade, Njegoševa 12, 11000 Belgrade, Serbia \\ ${ }^{3}$ Department of Physics, Concordia University, 7141 Sherbrooke Ouest, Montréal, Québec H4B 1R6, Canada
}

\author{
${ }^{a)}$ Author to whom correspondence should be addressed: m.tahir06@alumni.imperial.ac.uk \\ b) predrag222@gmail.com \\ c)p.vasilopoulos@concordia.ca
}

\begin{abstract}
The multilayer band structure of black phosphorus (BP) is highly anisotropic, and its bandgap is tunable by applying a perpendicular electric field $E_{z}$. Within a linear response theory, we study dc and ac transport in few-layer BP, as functions of the Fermi energy, temperature, or frequency, in the presence of a Zeeman field. The current response to an in-plane electric field along two perpendicular directions is anisotropic and reflects that of the energy spectrum. In addition, we study the Hall conductivity and power absorption spectrum. The Hall conductivity vanishes, but the power spectrum $P(\omega)$ shows a considerable structure as a function of the normalized frequency $\alpha=\hbar \omega / 2 \Delta$ and/or of the bandgap $2 \Delta$ tuned by $E_{z}$. In particular, a clear maximum occurs in the difference between spin-up and spin-down contributions to $P(\omega)$. When potassium $(\mathrm{K})$ atoms are deposited on top of $\mathrm{BP}$, the anisotropy in the current response is stronger and the power spectrum is further modified. The results may be pertinent to the development of phosphorene-based applications.
\end{abstract}

Published under license by AIP Publishing. https://doi.org/10.1063/1.5129273

\section{INTRODUCTION}

Phosphorene, a monolayer of black phosphorus (BP), has attracted great attention among two-dimensional (2D) materials ${ }^{1}$ due to its puckered and highly anisotropic electronic band structure. $^{2-4}$ It exhibits promising carrier mobilities, ${ }^{5}$ a direct bandgap of $2.0 \mathrm{eV}$, and unique anisotropic mechanical and transport properties useful for device applications. Many experimental works are devoted to its material growth, its physical characterization, and device explorations. ${ }^{2,3,7,8}$ There are several theoretical investigations dealing with its electronic band structure, ${ }^{9} \mathrm{dc}$ magnetotransport in the presence of periodic modulations, ${ }^{10}$ its anisotropic optical properties, ${ }^{11,12}$ plasmons, ${ }^{13}$ topological and edge states, ${ }^{14}$ strain-induced topological phase transitions, ${ }^{15}$ anisotropic composite fermions, ${ }^{16}$ electron-substrate phonon coupling, ${ }^{17}$ and the tuning of its bandgap by an electric field. ${ }^{18}$ Moreover, transport properties have also been studied in bilayer phosphorene and show a bandgap tuning due to the layered structure. ${ }^{19,20}$

Recently, there has been a great interest toward multilayer $\mathrm{BP}^{21-31}$ in which the gap decreases upon increasing the number of layers owing to the relatively strong van der Waals interactions between them but remains direct. In particular, few-layer BP possesses a unique band structure, with a dispersion nearly linear along the armchair direction but parabolic along the zigzag direction. $^{32,33}$ This unique structure leads to the expectation that by reducing the bandgap, novel features may result, e.g., in the formation of Dirac cones as in semimetallic graphene. By creating a fewlayer BP structure (either 4 layers or around 10-20 layers), the gap has been significantly reduced to $300 \mathrm{meV}^{34,35}$ Moreover, by doping or applying a perpendicular electric field to thick multilayer $\mathrm{BP},{ }^{34,35}$ the bandgap can be reduced to $50 \mathrm{meV}$ or vanish. Indeed, the electrostatic potential and charge distribution across a BP sample are controlled by the energetic balance between the induced interlayer capacitance and kinetic energy terms, which strongly depend on the thickness of the sample. ${ }^{36-38}$ This makes BP a promising candidate for electronic ${ }^{25-30}$ and optical applications. ${ }^{39-44}$ Furthermore, the multilayer BP crystal structure is highly anisotropic and gives rise to phenomena such as anisotropic electronic and thermal transport, ${ }^{45-47}$ linear dichroism, ${ }^{25,45}$ and anisotropic plasmons. ${ }^{37}$ Additionally, owing to its heavily puckered structure, multilayer BP is highly tunable by $\operatorname{strain}^{27,48}$ and electric field. ${ }^{34,49}$ Moreover, field-effect transistors, the quantum Hall effect, and related transport properties have been 
experimentally studied in the presence of a finite or zero magnetic. ${ }^{50-59}$ However, to our knowledge, transport properties of multilayer BP have not yet been considered; in our opinion, their study should be undertaken.

In this work, we consider multilayer BP in the presence of a Zeeman field, which creates significant spin splittings. Recently, an enhanced g-factor $(5.7 \pm 0.7)$ and large Zeeman splittings have been experimentally realized in multilayer $\mathrm{BP} .{ }^{59}$ In some detail, we analytically and numerically evaluate the $\mathrm{dc}$ and ac conductivities of such a system in the framework of a linear response theory. ${ }^{60,61}$ In addition, we evaluate the Hall conductivity and power absorption spectrum and assess their dependence on the Zeeman field and bandgap. The basic expressions are given in Sec. II and numerical results in Sec. III. Section V deals with BP when potassium (K) atoms are deposited on its top. A summary follows in Sec. VI.

\section{FORMALISM}

\section{A. Energy spectrum and eigenfunctions}

Multilayer BP is a highly anisotropic material with different properties along the $x$ and $y$ directions due to its puckered structure. The one-electron Hamiltonian has the form

$$
H_{0}=\left(\begin{array}{cc}
0 & \Delta+\frac{\hbar^{2} k_{x}^{2}}{2 m^{t}}-i \hbar v_{F} k_{y} \\
\Delta+\frac{\hbar^{2} k_{x}^{2}}{2 m^{*}}+i \hbar v_{F} k_{y} & 0
\end{array}\right),
$$

where $^{38} \Delta=0.18 \mathrm{eV}$ is the mass term that gives rise to a bandgap of $2 \Delta$. Furthermore, $m^{*}=1.42 m_{e}$ is the electron mass in the $x$ direction and $v_{F}=5.6 \times 10^{5} \mathrm{~m} / \mathrm{s}$ is the Fermi velocity along the $y$ direction. ${ }^{38}$ Including the Zeeman field, the total Hamiltonian reads

$$
H=H_{0}+s_{z} \Delta_{z} I_{2}
$$

where $I_{2}$ is the $2 \times 2$ identity matrix. The Zeeman term can be induced by a ferromagnetic substrate or by appropriate doping. Moreover, recently, an enhanced g-factor $(5.7 \pm 0.7)$ and large Zeeman splittings have been experimentally realized recently ${ }^{59}$ in multilayer BP. The value of Zeeman splitting for $B=35 \mathrm{~T}$ (used in Ref. 59) is $\Delta_{z}=10 \mathrm{meV}$, but we will use the value $\Delta_{z}=50 \mathrm{meV}$ throughout this work since, by using an appropriate substrate such as $\mathrm{EuO}$, one can achieve Zeeman field values in the range of $14 \mathrm{meV}-184 \mathrm{meV} .^{62}$ The eigenvalues of the Hamiltonian (2) are

$$
E_{\gamma, \mathbf{k}, s_{z}}=s_{z} \Delta_{z}+\gamma\left[\left(\Delta+\hbar^{2} k_{x}^{2} / 2 m^{*}\right)^{2}+\hbar^{2} v_{F}^{2} k_{y}^{2}\right]^{1 / 2} .
$$

Here, $\gamma=1(\gamma=-1)$ is for the conduction (valence) band. The corresponding eigenfunctions are

$$
\psi=\frac{e^{i\left(k_{x} x+k_{y} y\right)}}{\sqrt{2 L_{x} L_{y}}}\left(\begin{array}{c}
1 \\
\gamma e^{i \varphi}
\end{array}\right),
$$

where $\tan \varphi=\hbar v_{F} k_{y} /\left[\Delta+\hbar^{2} k_{x}^{2} /\left(2 m^{*}\right)\right]$.

In Fig. 1 , we show the energy dispersion vs momentum along the $x$ direction $\left(k_{y}=0\right)$ in (a) and the $y\left(k_{x}=0\right)$ one in (b). Along
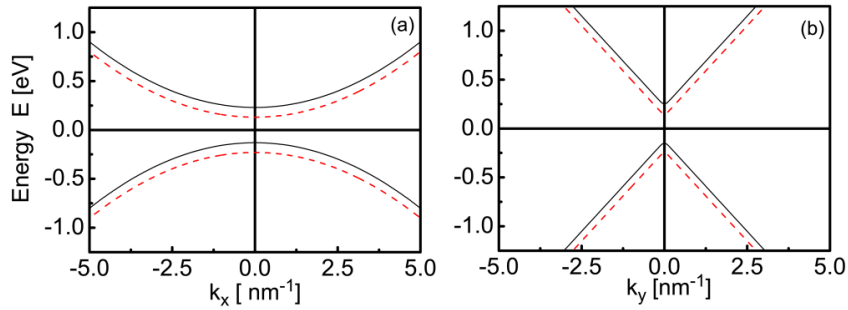

FIG. 1. Energy dispersion vs momentum along the $x$ direction in (a) and the $y$ one in (b). The solid (dashed) curves correspond to spin-up (spin-down) electrons.

the $x$ direction, the energy dispersion is parabolic, while along the $y$ one, it is linear. At a certain momentum, $k_{c}=1.3 \times 10^{10} \mathrm{~m}^{-1}$, they coincide, that is, when $E\left(k_{c}, 0\right)=E\left(0, k_{c}\right)$. The solid black curve corresponds to spin-up electrons, while the dashed one to spin-down electrons.

The density of states $D(E)$ is obtained from $D(E)=\sum_{\zeta} \delta\left(E-E_{\zeta}\right) \propto \int \delta\left(E-E_{\zeta}\right) d k_{x} d k_{y}$. The integration over $k_{y}$ can be carried out analytically, but the one over $k_{x}$ involves the elliptic integral $F(z)$ and is cumbersome. To avoid that, we evaluate $D(E)$ numerically and show it in Fig. 2. The solid black curve is for spin-up electrons and the red dotted one for spin-down electrons. It can be seen that the abrupt change occurs at $E_{ \pm}=\Delta \pm \Delta_{z}$.

\section{B. Conductivities}

We adopt the Kubo-type formulas of Ref. 60, which are valid for weak electric fields and weak scattering. The conductivity tensor has a diagonal and nondiagonal part, $\sigma_{\mu v}=\sigma_{\mu \nu}^{d}+\sigma_{\mu v}^{n d}$. In the absence of a magnetic field and for elastic scattering, the part $\sigma_{\mu v}^{d}$ is given by ${ }^{60}$

$$
\sigma_{\mu \nu}^{d}(i \omega)=\frac{\beta e^{2}}{S} \sum_{\zeta} f_{\zeta}\left(1-f_{\zeta}\right) \frac{v_{\nu \zeta} v_{\mu \zeta} \tau_{\zeta}}{1+i \omega \tau_{\zeta}},
$$

where $\omega$ is the frequency, $\tau_{\zeta}$ the relaxation time, and $v_{\mu \zeta}$ the diagonal matrix element of the velocity operator, $v_{\mu \zeta}=\left\langle\zeta\left|v_{\mu}\right| \zeta\right\rangle$

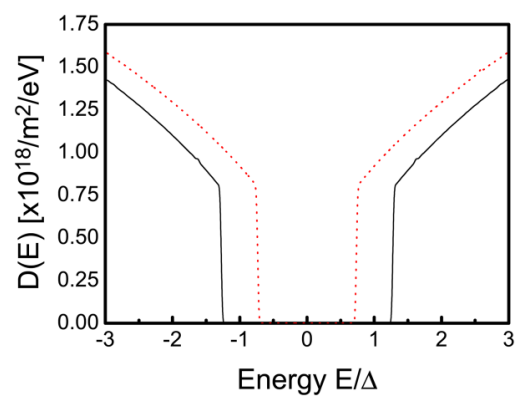

FIG. 2. Density of states as a function of the normalized energy $E / \Delta$, for spin-up (solid black curve) and spin-down (red dotted curve) electrons. 
$(\mu=x, y)$, with $|\zeta\rangle=\left|\gamma, s_{z}, \mathbf{k}\right\rangle$ and $S$ the area of the system. Furthermore, $f_{\zeta}$ is the Fermi-Dirac distribution, $\beta=1 / k_{B} T$, and $T$ the temperature.

The velocity operators are $v_{x}=\hbar k_{x} / m^{*} \sigma_{x}$ and $v_{y}=v_{F} \sigma_{y}$, where $\sigma_{x}$ and $\sigma_{y}$ are the Pauli matrices. We find that

$$
\begin{gathered}
v_{x \zeta}=\gamma_{\zeta}\left(\hbar k_{x} / m^{*}\right) \cos \varphi \\
v_{y \zeta}=\gamma_{\zeta} v_{F} \sin \varphi .
\end{gathered}
$$

The relaxation time $\tau_{\zeta}$ is given by

$$
\frac{1}{\tau_{\zeta}}=\frac{2 \pi n_{i}}{\hbar} \sum_{\zeta^{\prime}}\left|\left\langle\zeta|U(\mathbf{r})| \zeta^{\prime}\right\rangle\right|^{2} \delta\left(E_{\zeta}-E_{\zeta^{\prime}}\right)(1-\cos v),
$$

where $n_{i}$ is the concentration of impurities, $U(\mathbf{r})$ their potential, and $v$ the angle between the initial $(\mathbf{k})$ and final $\left(\mathbf{k}^{\prime}\right)$ electron wave vectors. We have chosen a moderate value for the impurity concentration $n_{i}=10^{8} \mathrm{~cm}^{-2}$; the corresponding time relaxation is on the order of $10^{-14} \mathrm{~s}$.

Using Eqs. (5)-(7), we can evaluate the conductivities $\sigma_{x x}$ and $\sigma_{y y}$. Relatively simple results are obtained for low temperatures, allowing the approximation $\beta f_{\zeta}\left(1-f_{\zeta}\right) \approx \delta\left(E_{\zeta}-E_{F}\right)$ and constant relaxation time $\tau_{\zeta} \equiv \tau$. Then, for $\mu=v$, Eq. (5) gives

$$
\sigma_{\mu \mu}^{d}(i \omega)=\frac{e^{2}}{S} \frac{\tau}{1+i \omega \tau} \sum_{\zeta} \delta\left(E_{\zeta}-E_{F}\right) v_{\mu \zeta}^{2}
$$

If $\tau$ is not taken as constant but is evaluated from Eq. (8), the result becomes unwieldy. We resort to a numerical evaluation of Eq. (9) with $\tau_{\zeta}$ obtained from Eq. (8). Notice though that, whether we use Eq. (5) for $\mu=v$ or Eq. (9), on account of Eqs. (6) and (7), we will have $\sigma_{x x} \neq \sigma_{y y}$; that is, the current response is asymmetric with respect to the $x$ and $y$ directions. Also, for simplicity, we neglect the difference in the effective mass of the conduction and valence bands because it is very small. ${ }^{38}$ We do so in all results and use the value $m^{*}=1.42 m_{e}$.

As far as the contribution $\sigma_{\mu \nu}^{n d}$ is concerned, one can cast the original expression ${ }^{60}$ in the familiar form as ${ }^{66}$

$$
\sigma_{\mu \nu}^{n d}(i \omega)=\frac{i \hbar e^{2}}{S} \sum_{\zeta \zeta^{\prime}} \frac{\left(f_{\zeta}-f_{\zeta^{\prime}}\right) v_{\nu \zeta \zeta^{\prime}} v_{\mu \zeta^{\prime} \zeta}}{\left(E_{\zeta}-E_{\zeta^{\prime}}\right)\left(E_{\zeta}-E_{\zeta^{\prime}}+\hbar \omega-i \eta\right)},
$$

where $v_{v \zeta \zeta^{\prime}}=\left\langle\zeta\left|v_{v}\right| \zeta^{\prime}\right\rangle$ and $v_{\mu \zeta^{\prime} \zeta}=\left\langle\zeta^{\prime}\left|v_{\mu}\right| \zeta\right\rangle$ are the off-diagonal matrix elements of the velocity operators with $v, \mu=x, y$ and $|\zeta\rangle \neq\left|\zeta^{\prime}\right\rangle$. From now, we replace the infinitesimal quantity $\eta$ in Eq. (10) by $\Gamma_{\zeta}$ in order to account for the broadening of the energy levels. ${ }^{58}$ The corresponding off-diagonal matrix elements are

$$
\begin{gathered}
v_{x \zeta \zeta^{\prime}}=\left(\hbar k_{x} / 2 m^{*}\right)\left(\gamma_{\zeta} e^{-i \varphi_{\zeta}}+\gamma_{\zeta^{\prime}} e^{i \varphi_{\zeta^{\prime}}}\right) \delta_{\mathbf{k}, \mathbf{k}^{\prime}}, \\
v_{y \zeta^{\prime} \zeta}=\left(i v_{F} / 2\right)\left(\gamma_{\zeta^{\prime}} e^{-i \varphi_{\zeta^{\prime}}}-\gamma_{\zeta} e^{i \varphi_{\zeta}}\right) \delta_{\mathbf{k}, \mathbf{k}^{\prime}},
\end{gathered}
$$

with $\zeta^{\prime} \neq \zeta$. This describes interband transitions only.
Using Eqs. (10)-(12), we obtain the intermediate result as

$$
\sigma_{y x}^{n d}(i \omega)=\frac{\hbar^{2} e^{2} v_{F}}{2 m^{*} S} \sum_{\zeta \neq \zeta^{\prime}} \frac{\left(f_{\zeta}-f_{\zeta^{\prime}}\right) k_{x} \gamma_{\zeta} \gamma_{\zeta^{\prime}} \sin \left(2 \varphi_{\zeta}\right)}{\left(E_{\zeta}-E_{\zeta^{\prime}}\right)\left(E_{\zeta}-E_{\zeta^{\prime}}+\hbar \omega-i \eta\right)} .
$$

The integral over $k_{x}$ in Eq. (13) vanishes because the integrand is an odd function of $k_{x}\left(\varphi_{\zeta}\right.$ is an even function of $k_{x}$ ). Accordingly, $\sigma_{y x}^{n d}$ vanishes identically.

\section{NUMERICAL RESULTS}

Below, we present numerical results for the conductivities given by Eqs. (5)-(8) and (10). The main reason is that the integrations over $k_{x}$ and $k_{y}$ lead to very cumbersome results involving elliptic integrals even in the simplest case of Eq. (9).

\section{A. dc transport}

First, we present numerical results for the diagonal part $\sigma_{x x}^{d}$ [panel (a)] and $\sigma_{y y}^{d}$ [panel (b)] according to Eq. (5) in Fig. 3 as a function of the normalized Fermi energy $\epsilon_{F}=E_{F} / \Delta$ assuming the Zeeman field $\Delta_{z}=50 \mathrm{meV}$ and $T=50 \mathrm{~K}$ (solid black curve). The red dashed curve corresponds to smaller $\Delta=0.1 \mathrm{eV}$ while keeping the Zeeman field unchanged. It can be seen that the conductivities $\sigma_{x x}^{d}$ and $\sigma_{y y}^{d}$ decrease monotonically when the Fermi level $E_{F}$ is in the valence band, then it vanishes for $E_{F}$ in the bandgap since there are no states to contribute to it, and finally rise again when $E_{F}$ is in the conduction band.

As for the components $\sigma_{x x}^{n d}, \sigma_{y y}^{n d}$, we do not show them because for $\omega=0$ and $\mu=v$, one can interchange the indices $\zeta$ and $\zeta^{\prime}$ in Eq. (10) and take half the sum. Then, one finds $\sigma_{x x}^{n d}(0)=0$ for $\eta=0$ or $\sigma_{x x}^{n d}(0) \ll \sigma_{x x}^{d}(0)$ for $\eta \approx 0$. The same holds for the component $\sigma_{y y}^{n d}$.

Next, in Fig. 4, we present results for the spin difference in the diagonal conductivity $\Delta \sigma_{i i}^{d}=\sigma_{i i}^{d}\left(s_{z}=+1\right)-\sigma_{i i}^{d}\left(s_{z}=-1\right)$, $(i=x, y)$ vs the normalized Fermi energy $\epsilon_{F}=E_{F} / \Delta$. The blue dashed and red dotted curves correspond to spin-up and spin-down components, respectively. As can be seen from the figures, the spin difference is large for negative values of $\epsilon_{F}$, then it becomes zero in the bandgap region (since there are no available states to contribute to it), and then it reaches large negative values for positive $\epsilon_{F}$. The reason for the negative values of $\Delta \sigma_{i i}^{d}$ for positive $\epsilon_{F}$ is the fact that
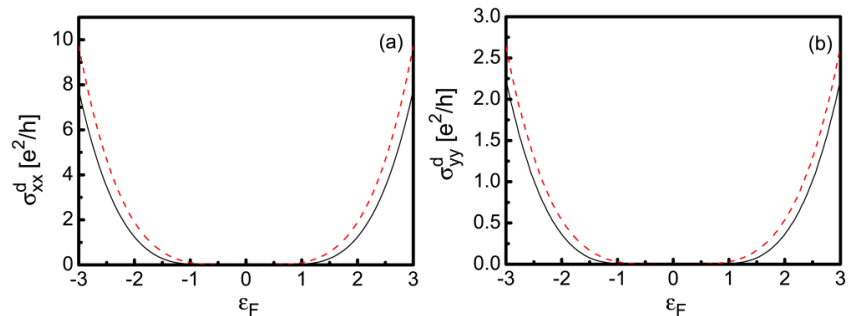

FIG. 3. The diagonal conductivity $\sigma_{x x}^{d}$ (a) and $\sigma_{y y}^{d}$ (b) vs normalized Ferm energy $\epsilon_{F}=E_{F} / \Delta$, at $T=50 \mathrm{~K}$. The black solid curves are for $\Delta=0.18 \mathrm{eV}$ and the red dashed ones for smaller $\Delta=0.1 \mathrm{eV}$. 

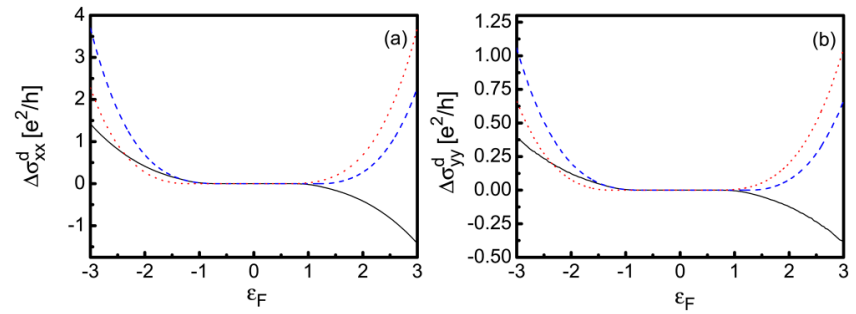

FIG. 4. The spin difference in the diagonal conductivity (solid black curve) $\Delta \sigma_{x x}^{d}$ (a) and $\Delta \sigma_{y y}^{d}$ (b) vs normalized Fermi energy $\epsilon_{F}=E_{F} / \Delta$, at $T=50 \mathrm{~K}$. The blue dashed (red dotted) curve corresponds to the spin-up (down) component.

$\sigma_{i i}^{d}\left(s_{z}=+1\right)$ is smaller than $\sigma_{i i}^{d}\left(s_{z}=-1\right)$, and the spin-up subband is shifted upward and becomes less populated. Notice that the amplitude in Fig. 4(a), when not vanishing, is approximately three times larger than that in Fig. 4(b). The amplitudes in Figs. 3(a) and 3(b) show a similar behavior.

\section{B. ac transport}

In this section, we evaluate the conductivities for finite frequency, level broadening, and temperature. We assume that the level broadening $\Gamma_{\zeta}$ is the same for all levels, i.e., $\Gamma_{\zeta} \approx \Gamma^{58}$

In Fig. 5(a), we show the real part of the diagonal part of the ac conductivity $\sigma_{x x}^{d}(\omega)$, given by Eq. (5), and in Fig. 5(b) the nondiagonal part, $\sigma_{x x}^{n d}(i \omega)$, obtained from Eq. (10), as functions of the normalized frequency $\alpha=\hbar \omega / 2 \Delta$. The solid curves are for $\Gamma=20 \mathrm{meV}$ and the dotted ones for $\Gamma=1 \mathrm{meV}$. It can be seen that $\sigma_{x x}^{d}$ monotonically decreases in agreement with the denominator in Eq. (5), while in Fig. 5(b), $\sigma_{x x}^{n d}(\omega)$ increases rapidly after $\alpha_{0}=2$. Notice also that the cusps in Fig. 5(b) are more noticeable for weaker disorder $\Gamma=1 \mathrm{meV}$.

Next, in Fig. 6(a), we show the real part of the diagonal part of the ac conductivity $\sigma_{y y}^{d}(\omega)$, given by Eq. (5), and in Fig. 6(b) the nondiagonal part, $\sigma_{y y}^{n d}(i \omega)$, given by Eq. (10). It can be seen that $\sigma_{y y}^{d}$ monotonically decreases in agreement with the denominator in Eq. (5), while in Fig. 6(b), $\sigma_{y y}^{n d}(\omega)$ shows two peaks at $\alpha_{0} \approx 2.7$ and $\alpha_{0} \approx 4$ that are more clearly seen for the weaker disorder $\Gamma=1 \mathrm{meV}$. These peaks are associated with interband transitions
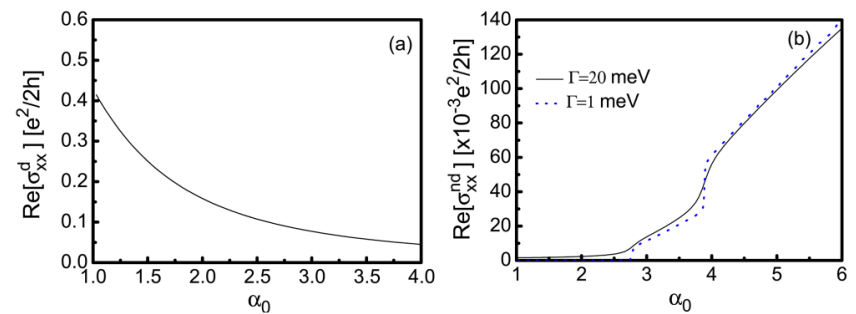

FIG. 5. The real parts of (a) $\sigma_{x x}^{d}(\omega)$ and (b) $\sigma_{x x}^{\text {nd }}(\omega)$ vs normalized frequency $\alpha=\hbar \omega / 2 \Delta$ for $E_{F}=0.3 \mathrm{eV}$. The solid curves are for $\Gamma=20 \mathrm{meV}$ and the dotted ones for $\Gamma=1 \mathrm{meV}$.
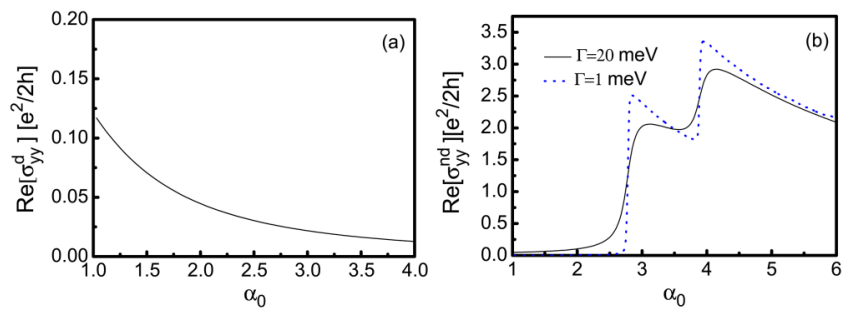

FIG. 6. As in Fig. 5 for $\sigma_{y y}^{d}(\omega)$ in (a) and $\sigma_{y y}^{n d}(\omega)$ in (b).

and are similar to those of Ref. 63 with $x$ and $y$ interchanged in line with its adopted Hamiltonian; see Figs. 4(a)-4(c) in Ref. 63. They do not occur in $\sigma_{x x}^{n d}(\omega)$ because the relevant transitions are forbidden. ${ }^{63,64}$ Notice though that in the dc limit, $\sigma_{y y}^{n d}(0)$ and $\sigma_{x x}^{n d}(0)$ do vanish only approximately; this is because we used a finite $\Gamma{ }^{58}$ see Sec. III A. We further notice that the scatteringdepending conductivities $\sigma_{y y}^{d}(i \omega)$ and $\sigma_{x x}^{d}(i \omega)$, which were not evaluated in Ref. 63, are the dominant ones for very low frequencies $\omega$.

\section{POWER SPECTRUM}

The average power absorbed from circularly polarized light of frequency $\omega$ and electric field $E$ (within linear response theory) is given by

$$
\begin{aligned}
P\left(\omega, s_{z}\right)= & \left(E^{2} / 2\right) \operatorname{Re}\left\{\sigma_{x x}\left(i \omega, s_{z}\right)+\sigma_{y y}\left(i \omega, s_{z}\right)\right. \\
& \left.-i \sigma_{x y}\left(i \omega, s_{z}\right)+i \sigma_{y x}\left(i \omega, s_{z}\right)\right\}
\end{aligned}
$$

We point out that $\sigma_{x y}^{n d}\left(i \omega, s_{z}\right)=\sigma_{y x}^{n d}\left(i \omega, s_{z}\right)=0$. We also have $\sigma_{y x}^{d}\left(i \omega, s_{z}\right)=\sigma_{x y}^{d}\left(i \omega, s_{z}\right)=0$; see Ref. 60 .

In Fig. 7 , we show the power spectrum as a function of the normalized frequency $\alpha_{0}$. The common parameters are level broadening $\Gamma=20 \mathrm{meV}^{58}$ and temperature $T=300 \mathrm{~K}$. Furthermore, $E_{F}=0.1 \mathrm{eV}$ for the solid and $E_{F}=0.3 \mathrm{eV}$ for the dashed curve.

We observe a maximum at $\alpha_{0}=2.25$ when the Fermi level lies in the bandgap $\left(E_{F}=0.1 \mathrm{eV}\right)$. Upon comparing with our similar work on $\mathrm{MoS}_{2}$ (Ref. 65), we see that in $\mathrm{MoS}_{2}$, we observed

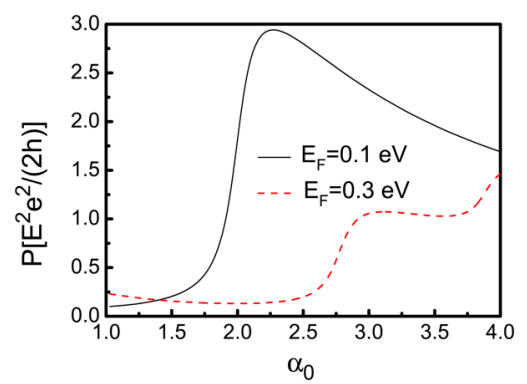

FIG. 7. Power spectrum vs normalized frequency $\alpha_{0}$ for two values of the Fermi energy: $E_{F}=0.1 \mathrm{eV}$ (solid curve) and $E_{F}=0.3 \mathrm{eV}$ (dashed curve). The temperature is $T=300 \mathrm{~K}$. 

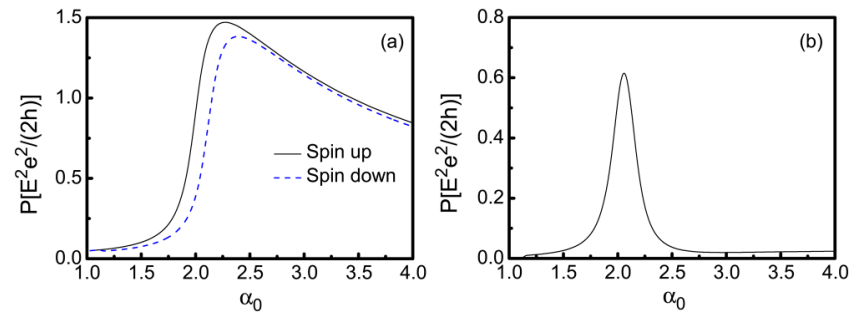

FIG. 8. (a) Spin-up (solid curve) and spin-down (dashed curve) contributions to the power spectrum vs normalized frequency $\alpha_{0}$ for $E_{F}=0.1 \mathrm{eV}$. The Zeeman field is $\Delta_{z}=50 \mathrm{meV}$ and the temperature is $T=300 \mathrm{~K}$. (b) Difference between the two contributions of (a) vs normalized frequency $\alpha_{0}$.

a dip between $\alpha_{0}=0$ and $\alpha_{0}=2$, whereas here, we have a dip until $\alpha_{0}=1.75$. The maximum at $\alpha_{0}=2.25$ occurs because the denominator in Eq. (10) approaches zero whenever $\hbar \omega \approx E_{c}-E_{v} \approx 2 \Delta+2 \Delta_{z}$. On the other hand, when the Fermi level is in the conduction band $\left(E_{F}=0.3 \mathrm{eV}\right)$, the maximum occurs later at $\alpha_{0}=3.0$.

We now consider the two spin directions separately and write

$$
P(\omega)=P\left(\omega, s_{z}=+1\right)+P\left(\omega, s_{z}=-1\right) .
$$

In Fig. 8(a), we show the spin components of the power spectrum vs normalized frequency for a Zeeman field $\Delta_{z}=50 \mathrm{meV}$. The solid curve shows the spin-up component and the dashed curve the spin-down one. The curves are shifted by an amount on the order of $2 \Delta_{z}$. In Fig. 8(b), we show the difference of the two components in (a), $\Delta P(\omega)=P\left(\omega, s_{z}=+1\right)-P\left(\omega, s_{z}=-1\right)$, vs normalized frequency. One can see a clear maximum at $\alpha_{0}=2.1$ approximately when the power spectrum has a maximum.

Finally, we investigate the effect of tuning the bandgap (by, e.g., $\left.E_{z}\right)$ on the power spectrum. In Fig. 9, we show the power spectrum vs $\Delta$ at fixed $\alpha_{0}=3.75$ and for two values of the Fermi level, $E_{F}=0.1 \mathrm{eV}$ (solid curve) and $E_{F}=0.3 \mathrm{eV}$ (dashed curve). It can be seen that the curve for $E_{F}=0.1 \mathrm{eV}$ decreases monotonically with $\Delta$, while the curve for $E_{F}=0.3 \mathrm{eV}$ exhibits a steplike behavior. Furthermore, after $\Delta \approx 0.23 \mathrm{eV}$, the two curves coincide.

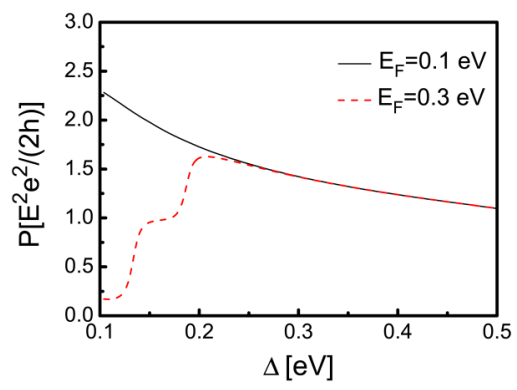

FIG. 9. Power spectrum vs mass term $\Delta$ at fixed $\alpha_{0}=3.75$ for $E_{F}=0.1 \mathrm{eV}$ (solid curve) and $E_{F}=0.3 \mathrm{eV}$ (dashed curve). The Zeeman field is $\Delta_{\mathrm{z}}=$ $50 \mathrm{meV}$ and the temperature is $T=300 \mathrm{~K}$.

\section{BP WITH POTASSIUM (K) DEPOSITED ON ITS TOP}

In this section, we investigate the electronic properties of fourlayer black phosphorus when potassium $(\mathrm{K})$ atoms are deposited on its top. When one introduces one $\mathrm{K}$ atom per $2 \times 2$ surface unit cell of four-layer BP, the band calculations of Ref. 38 show that the low-energy excitations near the Dirac point can be approximated, in terms of the Pauli matrices, by

$$
H^{\prime}=\hbar v_{F x} k_{x}^{\prime} \sigma_{x}+\hbar v_{F y} k_{y} \sigma_{y},
$$

where $v_{F x}=\hbar k_{D} / m^{*}$ is the velocity along the zigzag direction at the Dirac point, $v_{F x}=0.86 \times 10^{5} \mathrm{~m} / \mathrm{s}$, and $k_{x}^{\prime}=k_{x}-k_{D}$ is the value of $k_{x}$ with respect to the position $k_{D}$ of the Dirac point in the $k$ space. The bandgap is $\left|E_{g}\right|=\hbar^{2} k_{D}^{2} / m^{*}$. Furthermore, $v_{F y}=2.8 \times 10^{5} \mathrm{~m} / \mathrm{s}$ is the velocity along the armchair direction. The eigenvalues are

$$
E_{\gamma^{\prime}, \mathbf{k}, s_{z}}=s_{z} \Delta_{z}+\gamma^{\prime}\left[\left(\hbar v_{F x}\left(k_{x}-k_{D}\right)\right)^{2}+\left(\hbar v_{F y} k_{y}\right)^{2}\right]^{1 / 2} .
$$

Here, $\gamma^{\prime}=+1(-1)$ is for the conduction (valence) band. The corresponding eigenfunctions are

$$
\psi=\frac{e^{i\left(k_{x} x+k_{y} y\right)}}{\sqrt{2 L_{x} L_{y}}}\left(\begin{array}{c}
1 \\
\gamma^{\prime} e^{i \varphi^{\prime}}
\end{array}\right),
$$

with $\tan \varphi^{\prime}=\hbar v_{F y} k_{y} /\left(\hbar v_{F x}\left(k_{x}-k_{D}\right)\right)$. The velocity matrix elements are $v_{x \zeta}=\gamma^{\prime} v_{F x} \cos \left(\varphi^{\prime}\right) \delta_{\mathbf{k}, \mathbf{k}^{\prime}}$ and $v_{y \zeta}=\gamma^{\prime} v_{F y} \sin \left(\varphi^{\prime}\right) \delta_{\mathbf{k}, \mathbf{k}^{\prime}}$. In Figs. 10(a) and 10(b), we show the diagonal conductivity $\sigma_{x x}^{d}$ and $\sigma_{y y}^{d}$ vs normalized Fermi energy $\epsilon_{F}=2 E_{F} / E_{g}$, at $T=50 \mathrm{~K}$ for BP with deposited potassium $(\mathrm{K})$ on its top. It can be seen that the diagonal conductivity along the $x$ direction is of the same magnitude as for pristine four-layer BP, while that along the $y$ direction is considerably larger. The discrepancy along the $y$ direction with the pristine BP is due to a different angle $\varphi^{\prime}$ in the eigenfunctions.

Finally, we show (Fig. 11) the power spectrum vs the normalized frequency $\alpha_{0}$ for BP with deposited potassium (K) atoms on its top. The results are given for two values of the Fermi energy $E_{F}=0.1 \mathrm{eV}$ (solid black curve) and $E_{F}=0.3 \mathrm{eV}$ (red dashed curve). Comparing these results with those for pristine BP, shown in Fig. 7 , one can conclude that when the Fermi level is higher $\left(E_{F}=0.3 \mathrm{eV}\right)$, the values of the power spectrum are larger for
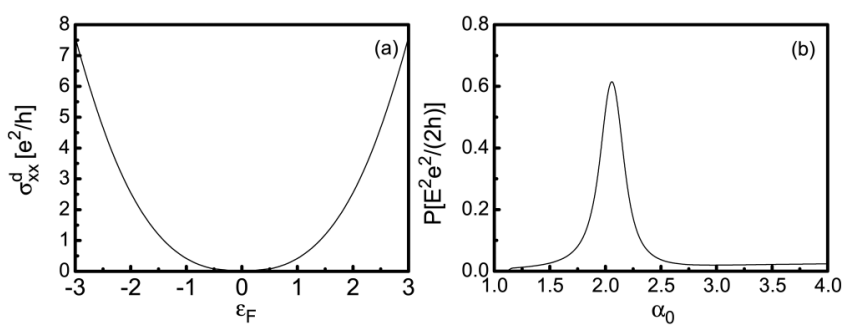

FIG. 10. The diagonal conductivity $\sigma_{x x}^{d}$ (a) and $\sigma_{y y}^{d}$ (b) vs normalized Fermi energy $\epsilon_{F}=2 E_{F} / E_{g}$, at $T=50 \mathrm{~K}$ for BP with potassium (K) atoms deposited on its top 


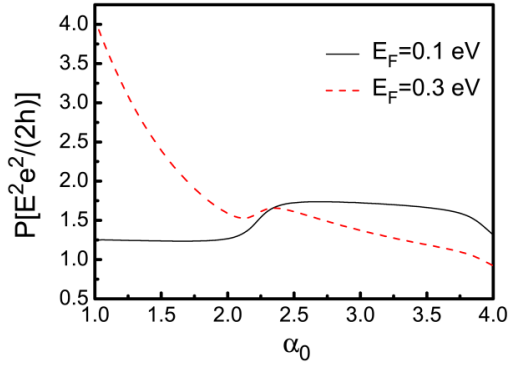

FIG. 11. Power spectrum vs normalized frequency $\alpha$ for $E_{F}=0.1 \mathrm{eV}$ (solid black curve) and $E_{F}=0.3 \mathrm{eV}$ (red dashed curve) for BP with potassium (K) atoms deposited on its top. The temperature is $T=300 \mathrm{~K}$.

smaller $\alpha_{0}$ and that the spectrum has a local minimum for $\alpha_{0}=3.0$. The initial large value for $E_{F}=0.3 \mathrm{eV}$ is due to the large conductivity $\sigma_{y y}^{d}$. On the other hand, when the Fermi level is in the bandgap, $E_{F}=0.1 \mathrm{eV}$, there is a flat region for $1.5<\alpha_{0}<3.5$, which is absent in pristine BP.

\section{SUMMARY}

We calculated the ac and dc conductivities of few-layer phosphorus within the linear response transport approach. Though not explicitly shown, we can affirm that the nondiagonal components $\sigma_{x x}^{n d}\left(i \omega, s_{z}\right)$ and $\sigma_{y y}^{n d}\left(i \omega, s_{z}\right)$ of the optical conductivity remain almost unaffected up to approximately $300 \mathrm{~K}$. This is because they depend very weakly on any interaction through the value of $\Gamma$. For the diagonal components $\sigma_{x x}^{d}\left(i \omega, s_{z}\right)$ and $\sigma_{y y}^{d}\left(i \omega, s_{z}\right)$ though, we cannot affirm that because at high temperatures, an electron-phonon interaction takes place, which was not taken into account. Notice that for the electron-impurity interaction treated here, the dc limit $(\omega=0)$ of $\sigma_{x x}^{n d}\left(i \omega, s_{z}\right)$ and $\sigma_{y y}^{n d}\left(i \omega, s_{z}\right)$ is almost zero, and the components $\sigma_{x x}^{d}\left(i \omega, s_{z}\right)$ and $\sigma_{y y}^{d}\left(i \omega, s_{z}\right)$ dominate. These contributions to the current were not evaluated in Ref. 63.

The power spectrum $P(\omega)$ depends on the mass term $\Delta$, tuned by the external electric field $E_{z}$, and slightly on the Zeeman field. Notice, however, that a clear maximum occurs in the difference between the spin-up and spin-down components of $P(\omega)$ when plotted vs the normalized frequency $\alpha$; cf. Fig. 8(b).

The above results can be contrasted with those on bilayer $\mathrm{WSe}_{2} .{ }^{66}$ Here, there is no spin or valley Hall transport, as in Ref. 66, since the relevant Hamiltonian terms are not present in Eq. (1). However, the power spectrum $P(\omega)$ vs $\omega$ shows a similar but somewhat simpler structure than in Ref. 66. On the other hand, the asymmetry of the spectrum (3) leads to results, e.g., those of Figs. 4, 6, and 10, which are absent in Ref. 66.

Finally, we contrasted the pristine BP results with those for a four-layer BP when potassium (K) atoms are deposited on its top. We found that the anisotropy in the current response is stronger, and the power spectrum is further modified in the latter case. We expect the results to be useful in the development of phosphorenebased applications.

\section{ACKNOWLEDGMENTS}

This work was supported by the Colorado State University (M.T.), the Ministry of Education, Science, and Technological Development of Serbia within Project No. TR 32008 (P.M.K.), and the Canadian NSERC under Grant No. OGP0121756 (P.V.).

\section{REFERENCES}

${ }^{1}$ X. Xu, W. Yao, D. Xiao, and T. F. Heinz, Nat. Phys. 10, 343 (2014).

${ }^{2}$ X. Ling, H. Wang, S. Huang, F. Xia, and M. S. Dresselhaus, Proc. Natl. Acad. Sci. U.S.A. 112, 4523 (2015).

${ }^{3}$ S. Z. Butler, S. M. Hollen, L. Cao, Y. Cui, J. A. Gupta, H. R. Gutierrez, T. F. Heinz, S. S. Hong, J. Huang, A. F. Ismach, E. J. Halperin, M. Kuno, V. V. Plashnitsa, R. D. Robinson, R. S. Ruoff, S. Salahuddin, J. Shan, L. Shi, M. G. Spencer, M. Terrones, W. Windl, and J. E. Goldberger, ACS Nano 7, 2898 (2013).

${ }^{4}$ L. Cartz, S. R. Srinivasa, R. J. Riedner, J. D. Jorgensen, and T. G. Worlton, J. Chem. Phys. 71, 1718 (1979).

${ }^{5}$ J. Qiao, X. Kong, Z.-X. Hu, F. Yang, and W. Ji, Nat. Commun. 5, 4475 (2014).

${ }^{6}$ L. Liang, J. Wang, W. Lin, B. G. Sumpter, V. Meunier, and M. Pan, Nano Lett. 14, 6400 (2014).

${ }^{7}$ R. J. Suess, M. M. Jadidi, T. E. Murphy, and M. Mittendorff, Appl. Phys. Lett. 107, 081103 (2015);H. Yuan, X. Liu, F. Afshinmanesh, W. Li, G. Xu, J. Sun, B. Lian, A. G. Curto, G. Ye, Y. Hikita, Z. Shen, S. Zhang, X. Chen, M. Brongersma, H. Y. Hwang, and Y. Cui, Nat. Nanotechnol. 10, 707 (2015).

${ }^{8}$ L. Li, F. Yang, G. J. Ye, Z. Zhang, Z. Zhu, W. Lou, X. Zhou, L. Li, K. Watanabe, T. Taniguchi, K. Chang, Y. Wang, X. H. Chen, and Y. Zhang, Nat. Nanotechnol. 11, 593 (2016).

${ }^{9}$ A. N. Rudenko and M. I. Katsnelson, Phys. Rev. B 89, 201408(R) (2014);P. Li and I. Appelbaum, ibid. 90, 115439 (2014);J. M. Pereira, Jr. and M. I. Katsnelson, ibid. 92, 075437 (2015).

${ }^{10}$ M. Tahir and P. Vasilopoulos, J. Phys.: Condens. Matter 29, 425302 (2017).

${ }^{11}$ T. Low, A. S. Rodin, A. Carvalho, Y. Jiang, H. Wang, F. Xia, and A. H. Castro Neto, Phys. Rev. B 90, 075434 (2014).

${ }^{12}$ X. Y. Zhou, R. Zhang, J. P. Sun, Y. L. Zou, D. Zhang, W. K. Lou, F. Cheng, G. H. Zhou, F. Zhai, and K. Chang, Sci. Rep. 5, 12295 (2015).

${ }^{13}$ A. Nemilentsau, T. Low, and G. Hanson, Phys. Rev. Lett. 116, 066804 (2016).

${ }^{14}$ R. Fei, Vy Tran, and Li Yang, Phys. Rev. B 91, 195319 (2015);B. Ostahie and A. Aldea, ibid. 93, 075408 (2016).

${ }^{15}$ E. T. Sisakht, F. Fazileh, M. H. Zare, M. Zarenia, and F. M. Peeters, Phys. Rev. B 94, 085417 (2016).

${ }^{16}$ A. Ghazaryan and T. Chakraborty, Phys. Rev. B 92, 165409 (2015); A. C. Balram and J. K. Jain, ibid. 93, 075121 (2016).

${ }^{17}$ A. Mogulkoc, Y. Mogulkoc, A. N. Rudenko, and M. I. Katsnelson, Phys. Rev. B 93, 085417 (2016).

${ }^{18}$ S. Yuan, E. v. Veen, M. I. Katsnelson, and R. Roldan, Phys. Rev. B 93, 245433 (2016).

${ }^{19}$ S. Yuan, A. N. Rudenko, and M. I. Katsnelson, Phys. Rev. B 91, 115436 (2015).

${ }^{20}$ G. Gaddemane, W. G. Vandenberghe, M. L. Van de Put, S. Chen, S. Tiwari, E. Chen, and M. V. Fischetti, Phys. Rev. B 98, 115416 (2018).

${ }^{21}$ L. Li, Y. Yu, G. J. Ye, Q. Ge, X. Ou, H. Wu, D. Feng, X. H. Chen, and Y. Zhang, Nat. Nanotechnol. 9, 372 (2014).

${ }^{22}$ H. Liu, A. T. Neal, Z. Zhu, Z. Luo, X. Xu, D. Tománek, and P. D. Ye, ACS Nano 8, 4033 (2014).

${ }^{23}$ W. Lu, H. Nan, J. Hong, Y. Chen, C. Zhu, Z. Liang, X. Ma, Z. Ni, C. Jin, and Z. Zhang, Nano Res. 7, 853 (2014).

${ }^{24}$ S. Das, W. Zhang, M. Demarteau, A. Hoffmann, M. Dubey, and A. Roelofs, Nano Lett. 14, 5733 (2014).

${ }^{25}$ F. Xia, H. Wang, and Y. Jia, Nat. Commun. 5, 5458 (2014).

${ }^{26}$ V. Tran, R. Soklaski, Y. Liang, and L. Yang, Phys. Rev. B 89, 235319 (2014).

${ }^{27}$ R. Fei and L. Yang, Nano Lett. 14, 2884 (2014). 
${ }^{28}$ R. Fei, A. Faghaninia, R. Soklaski, J.-A. Yan, C. Lo, and L. Yang, Nano Lett. 14, 6393 (2014)

${ }^{29}$ X. Wang, A. M. Jones, K. L. Seyler, V. Tran, Y. Jia, H. Zhao, H. Wang, L. Yang, X. Xu, and F. Xia, Nat. Nanotechnol. 10, 517 (2015).

${ }^{30}$ Z. Zhu and D. Tomanek, Phys. Rev. Lett. 112, 176802 (2014).

${ }^{31}$ S. Park, S. Woo, and H. Min, 2D Mater. 6, 025016 (2019).

${ }^{32}$ V. Tran and L. Yang, Phys. Rev. B 89, 245407 (2014).

${ }^{33}$ A. S. Rodin, A. Carvalho, and A. H. Castro Neto, Phys. Rev. Lett. 112, 176801 (2014).

${ }^{34}$ B. Deng, V. Tran, Y. Xie, H. Jiang, C. Li, Q. Guo, X. Wang, H. Tian, S. J. Koester, H. Wang, J. J. Cha, Q. Xia, L. Yang, and F. Xia, Nat. Commun. 8, 14474 (2017).

${ }^{35}$ Y. Liu, Z. Qiu, A. Carvalho, Y. Bao, H. Xu, S. J. R. Tan, W. Liu, A. H. C. Neto, K. P. Loh, and J. Lu, Nano Lett. 17, 1970 (2017).

${ }^{36}$ Q. Liu, X. Zhang, L. B. Abdalla, A. Fazzio, and A. Zunger, Nano Lett. 15, 1222 (2015).

${ }^{37}$ T. Low, R. Roldán, H. Wang, F. Xia, P. Avouris, L. M. Moreno, and F. Guinea, Phys. Rev. Lett. 113, 106802 (2014).

${ }^{38}$ S. S. Baik, K. S. Kim, Y. Yi, and H. J. Choi, Nano Lett. 15, 7788 (2015).

${ }^{39}$ M. Tahir, P. Vasilopoulos, and F. M. Peeters, Phys. Rev. B 92, 045420 (2015).

${ }^{40}$ Y. Jing, Q. Tang, P. He, Z. Zhou, and P. Shen, Nanotechnology 26(9), 095201 (2015).

${ }^{41}$ M. Buscema, D. J. Groenendijk, S. I. Blanter, G. A. Steele, H. S. J. van der Zant, and A. Castellanos-Gomez, Nano. Lett. 14, 3347 (2014).

${ }^{42}$ S. P. Koenig, R. A. Doganov, H. Schmidt, A. H. C. Neto, and B. Oezyilmaz, Appl. Phys. Lett. 104, 103106 (2014).

${ }^{43}$ D. Çakír, C. Sevik, and F. M. Peeters, Phys. Rev. B 92, 165406 (2015).

${ }^{44}$ D. Çakír, H. Sahin, and F. M. Peeters, Phys. Rev. B 90, 205421 (2014).

${ }^{45}$ S. Yuan, A. Rudenko, and M. Katsnelson, Phys. Rev. B 91, 115436 (2015).

${ }^{46}$ G. Qin, Q.-B. Yan, Z. Qin, S.-Y Yue, M. Hu, and G. Su, Phys. Chem. Chem. Phys. 17, 4854 (2015).

${ }^{47}$ Y. Cai, Q. Ke, G. Zhang, Y. P. Feng, V. B. Shenoy, and Y.-W. Zhang, Adv. Funct. Mater. 25, 2230 (2015).

${ }^{48}$ M. Elahi, K. Khaliji, S. M. Tabatabaei, M. Pourfath, and R. Asgari, Phys. Rev. B 91, 115412 (2015).
${ }^{49}$ B. Jhun and C.-H. Park, Phys. Rev. B 96, 085412 (2017);L. L. Li, B. Partoens, and F. M. Peeters, ibid. 97, 155424 (2018).

${ }^{50} \mathrm{~N}$. Gillgren, D. Wickramaratne, Y. Shi, T. Espiritu, J. Yang, J. Hu, J. Wei, X. Liu, Z. Mao, K. Watanabe, T. Taniguchi, M. Bockrath, Y. Barlas, R. K. Lake, and C. N. Lau, 2D Mater. 2, 011001 (2014).

${ }^{51}$ X. Chen, Y. Wu, Z. Wu, Y. Han, S. Xu, L. Wang, W. Ye, T. Han, Y. He, Y. Cai, and N. Wang, Nat. Commun. 6, 7315 (2015).

${ }^{52}$ L. Li, G. J. Ye, V. Tran, R. Fei, G. Chen, H. Wang, J. Wang, K. Watanabe, T. Taniguchi, L. Yang, X. H. Chen, and Y. Zhang, Nat. Nanotechnol. 10, 608 (2015).

${ }^{\mathbf{5 3}}$ G. Long, S. Xu, J. Shen, J. Hou, Z. Wu, T. Han, J. Lin, W. K. Wong, Y. Cai, R. Lortz, and N. Wang, 2D Mater. 3, 031001 (2016).

${ }^{54}$ V. Tayari, N. Hemsworth, I. Fakih, A. Favron, E. Gaufres, G. Gervais, R. Martel, and T. Szkopek, Nat. Commun. 6, 7702 (2015).

${ }^{55}$ L. Li, F. Yang, G. J. Ye, Z. Zhang, Z. Zhu, W. Lou, X. Zhou, L. Li, K. Watanabe, T. Taniguchi, K. Chang, Y. Wang, X. H. Chen, and Y. Zhang, Nat. Nanotechnol. 11, 593 (2016).

${ }^{56}$ G. Long, D. Maryenko, J. Shen, S. Xu, J. Hou, Z. Wu, W. K. Wong, T. Han, J. Lin, Y. Cai, R. Lortz, and N. Wang, Nano Lett. 16, 7768 (2016).

${ }^{57}$ G. Long, D. Maryenko, S. Pezzini, S. Xu, Z. Wu, T. Han, J. Lin, C. Cheng, Y. Cai, U. Zeitler, and N. Wing, Phys. Rev. B 96, 155448 (2017).

${ }^{58}$ J. Yang, S. Tran, J. Wu, S. Che, P. Stepanov, T. Taniguchi, K. Watanabe, H. Baek, D. Smirnov, R. Chen, and C. N. Lau, Nano Lett. 18, 229 (2018).

${ }^{59}$ F. Yang, Z. Zhang, N. Z. Wang, G. J. Ye, W. Lou, X. Zhou, K. Watanabe, T. Taniguchi, K. Chang, X. H. Chen, and Y. Zhang, Nano Lett. 18, 6611 (2018).

${ }^{60}$ M. Charbonneau, K. M. Van Vliet, and P. Vasilopoulos, J. Math. Phys. 23, 318 (1982).

${ }^{61}$ V. Vargiamidis, P. Vasilopoulos, and G.-Q. Hai, J. Phys.: Condens. Matter 26, 345303 (2014)

${ }^{62}$ H. Chen, B. Li, and J. Yang, ACS Appl. Mater. Interfaces 9, 38999 (2017).

${ }^{63}$ J. Jang, S. Ahn, and H. Min, 2D Mater. 6, 025029 (2019).

${ }^{64}$ H. Yuan, X. Liu, F. Afshinmanesh, W. Li, G. Xu, J. Sun, B. Lian, A. G. Curto, G. Ye, and Y. Hikita, Nat. Nanotechnol. 10, 707 (2015).

${ }^{65}$ P. M. Krstajić, M. Tahir, and P. Vasilopoulos, Phys. Rev. B 94, 085413 (2016).

${ }^{66}$ M. Tahir, P. M. Krstajić, and P. Vasilopoulos, Phys. Rev. B 98, 075429 (2018). 